\title{
Speed control of high speed aircraft with fixed attack angle and constant elastic shape states

\author{
Yuqiang $\mathrm{Jin}^{1}$ and Hong Wang ${ }^{2}$
}

${ }^{1}$ Department of Training, Naval aeronautical and astronautical UniversityYanti, 264001

${ }^{2}$ Department of Control engineering, Naval aeronautical and astronautical University Yanti, 264001

ajinyuqiang1024@126.com

Keywords: aircraft; simulation; speed control; virtual flying; stability

\begin{abstract}
For high speed vehicles, the engine model is difficult to built. Also it is very important and difficult to design a proper oil supplying law to make the speed of control object to tracking the desired value as accurate as possible. So a kind of speed controller is designed based on the pitch channel model built according to Lagrange equation and released by USA force. Since the speed of this flying object is too high, to make the question simple, a kind of virtual flying condition is proposed such that the design of speed controller can be simple and easy to verify. So the attack angle is assumed to be constant and the elastic shape states are also assumed to be a constant. And this virtual flying design method can not only make the speed controller design simple, it can also make it is possible to testify the rightness of the engine model with the help of numerical simulation technology.
\end{abstract}

\section{Introduction}

The thrust model of hypersonic aircraft is very complex, scholars are attracted extensive research interest by the engine model ${ }^{[1-4]}$. The complex of force model mainly depends on a huge impact of attack angle and elastic form. The engine thrust model even can be used in place of constant in a digital simulation analysis of hypersonic flight vehicle ${ }^{[5-8]}$. In accordance with the research principle of from simple to complex, we extract the simple model of hypersonic engine, attack angle is constant elastic form in constant case. The relationship between the vehicle speed and engine oil supply factor is researched ${ }^{[9-12]}$. Through speed control research of a simple model, on the one hand we can preliminary verify the rationality of the engine, on the other hand we can preliminary understand the dynamic characteristics of engine, and the coupling relationship between attack angle and elastic form. At the same time, the simulation curve can image show the rationality of engine data and the complex nonlinear relation between attack angle and elastic form by simulation research of different constant attack angle and elasticity form.

\section{Model Description}

Considering the elastic shape structure, a kind of pitch channel hypersonic aircraft model built according to Lagrange equation is released by USA air force as followed:

$$
\begin{gathered}
\dot{V}=\frac{T \cos \alpha-D}{m}-g \sin \gamma \\
\dot{\phi}=-2 \varsigma \omega_{n} \phi-\omega_{n}^{2} \phi+\omega_{n}^{2} \phi_{c} \\
\dot{\gamma}=\frac{L+T \sin \alpha}{m V}-\frac{g \cos \gamma}{V} \\
\dot{\alpha}=q-\dot{\gamma} \\
\dot{q}=\frac{M}{I}
\end{gathered}
$$




$$
\begin{gathered}
\dot{h}=V \sin \gamma \\
\ddot{\eta}_{i}=-2 \varepsilon_{m} \omega_{m i} \dot{\eta}_{i}-\omega_{m i}^{2} \eta_{i}+N_{i}
\end{gathered}
$$

Where

$$
T=\bar{q} s\left(C_{T \phi} \phi+C_{T}+C_{T}^{\eta} \eta\right), D=\bar{q} S C_{D}
$$

And $V$ is speed, $\gamma$ is the speed angle, $\alpha$ is attack angle, $Q$ is the attitude angle speed, $h$ is the height. $\phi$ is the oil supplying factor, $\delta_{c}$ is the duck wing and $\delta_{e}$ is the lift rudder.

\section{Simulation settings of free flying}

In order to testify the rightness of the model of hypersonic aircraft, choose a initial height as $h_{0}=85000 * 0.3048$, initial speed as $V_{0}=7846 * 0.3048$, initial attack angle as $\alpha_{0}=0.0174$, and other initial state as: $\gamma_{0}=0, \quad q_{0}=0, \quad \eta_{10}=0.4588 * 0.3048 * 14.59, \quad \eta_{20}=-0.08726 * 0.3048 * 14.59$, $\eta_{30}=-0.03671 * 0.3048 * 14.59$, Setting the throttle opening of engine is the switching control law for the PID control law and diffusion control law. Selecting

$$
\phi_{c}=k_{e t} e_{v}+k_{s e t} e_{v}+k_{d e} \dot{e}_{v}+k_{e t b} \frac{e_{v}}{\left|e_{v}\right|+\varepsilon_{e v}}, e_{v}>0, \phi_{c}=-300, e_{v}<0
$$

Where $e_{v}=V^{d}-V, k_{e t} 、 k_{\text {set }} 、 k_{d e} 、 k_{e t b}$ and $\varepsilon_{e v}$ are the adjustable positive. Selecting that expectations speed is $V^{d}=2391+\Gamma^{*} 0.5 * 5 t^{2}$, and choose the control as $\delta_{c}=0$, then the free flying test can be done by numerical simulation to make $\dot{\alpha}=0, \dot{\eta}_{i}=0$ and simulation time is set as 15 second.

\section{Numerical Simulation and result analysis}

With above model and air coefficients and set initial condition as above paragraph , write a program with $\mathrm{m}$ language in Matlab software, then the simulation can be done and simulation results can be shown as following figures.

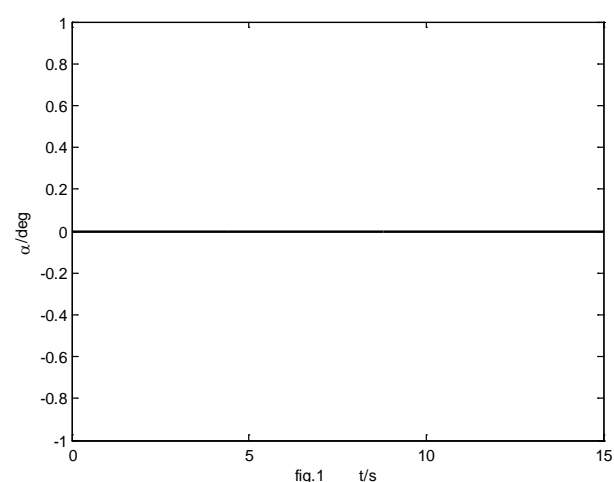

Fig 1 The curve of attack angle

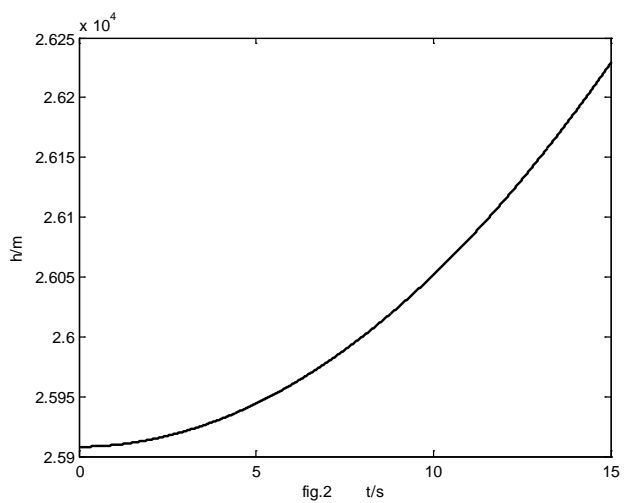

Fig 2 The curve of the height 


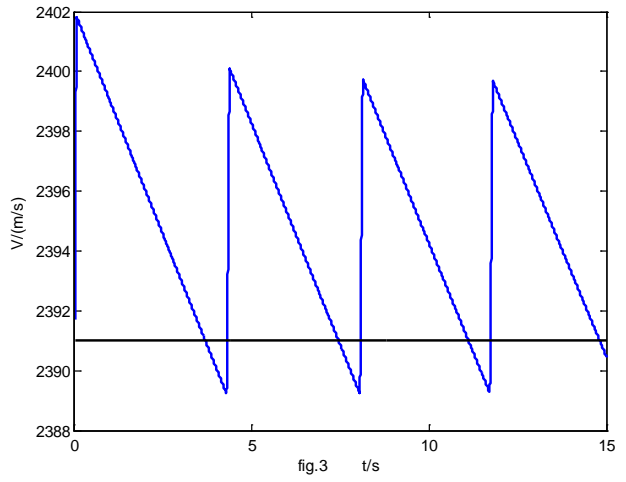

Fig 3 The curve of speed

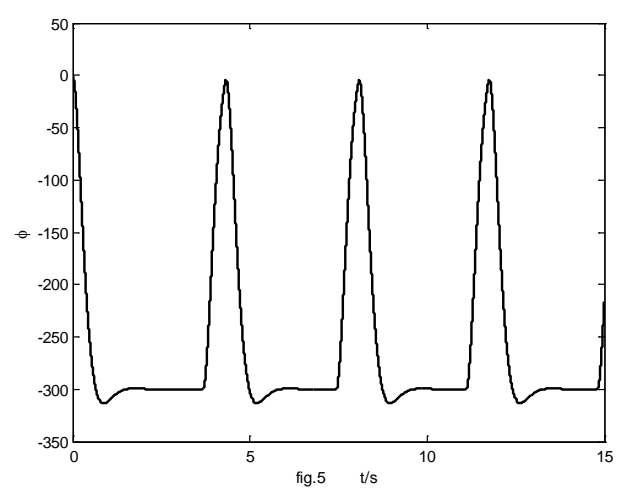

Fig 5 The curve of oil supplying factor

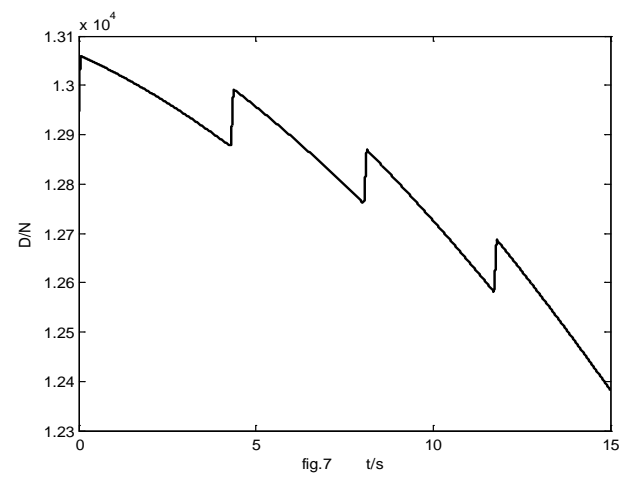

Fig 7 The curve of resistance

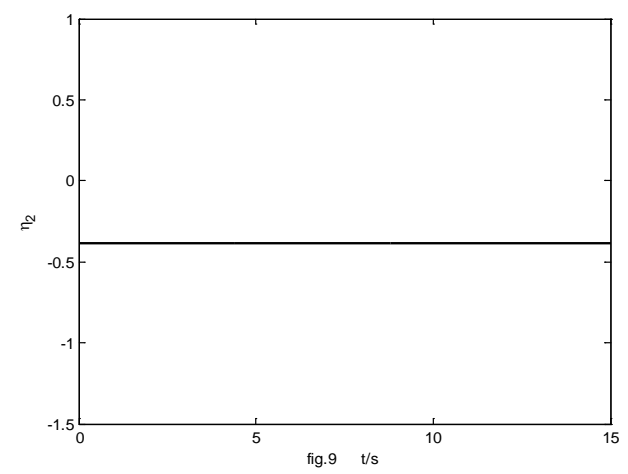

Fig 9 The state of second elastic shape

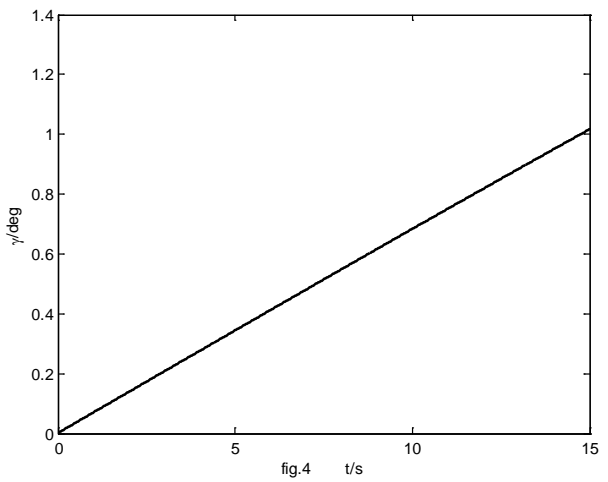

Fig 4 The curve of speed angle

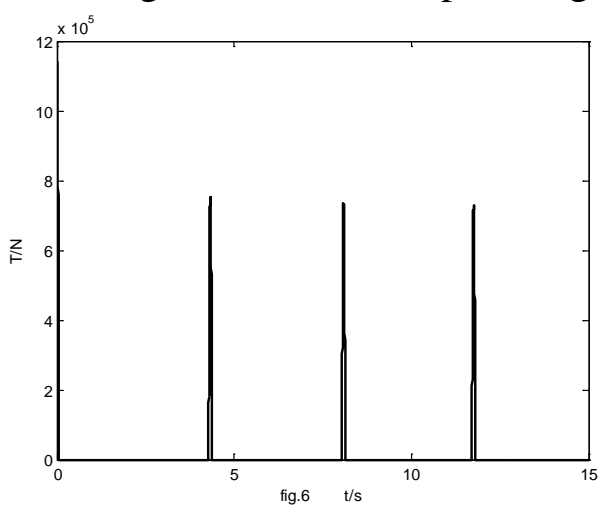

Fig 6 The curve of thrust

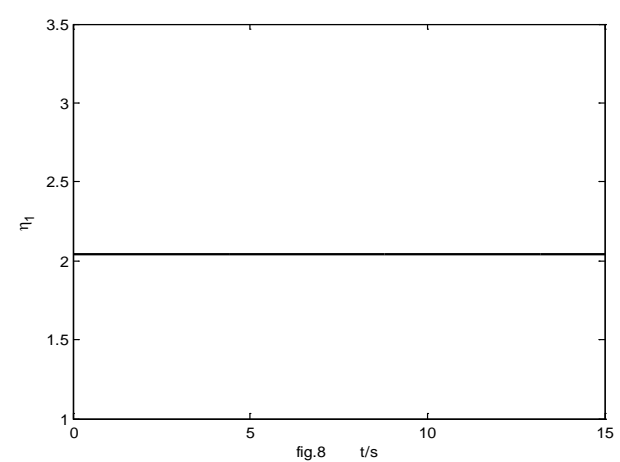

Fig 8 The state of first elastic shape

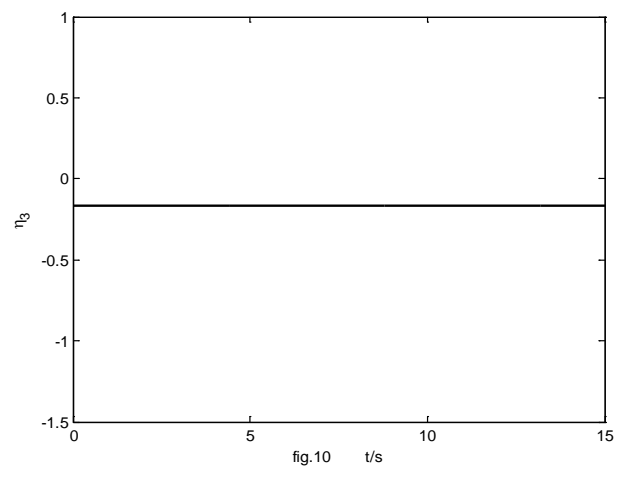

Fig 10 The state of third elastic shape 


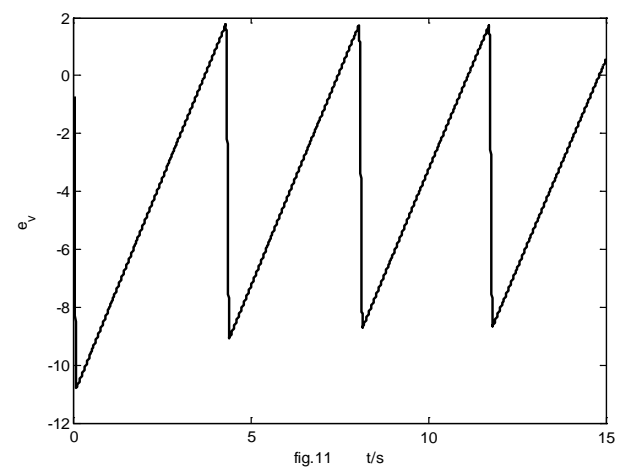

Fig 11 The state of speed error

It can be seen from simulation that the elevation angle keeps basic stable in the fixed attack angle and elastic 15 seconds flight. And aircraft speed can carry on the track of a given speed. The engine thrust keeps pulse type switch. The velocity error can be accurate to within $20 \mathrm{~m} / \mathrm{s}$, thus the speed control law is effective, setting $\Gamma=1$ as the variable accelerated flight test can also be considered. The simulation results are as follows:

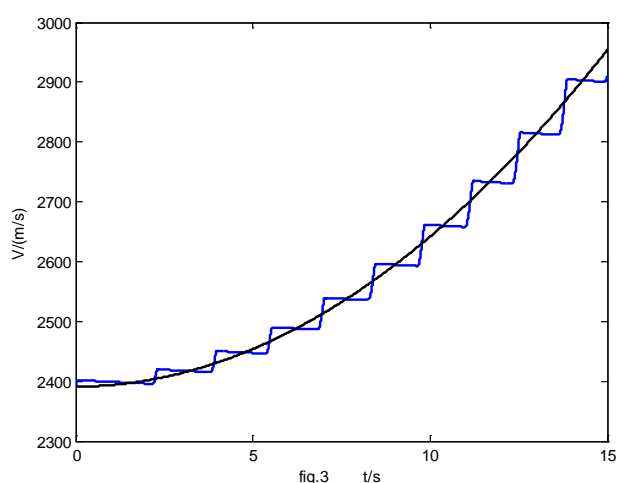

Fig 12 The state of speed error

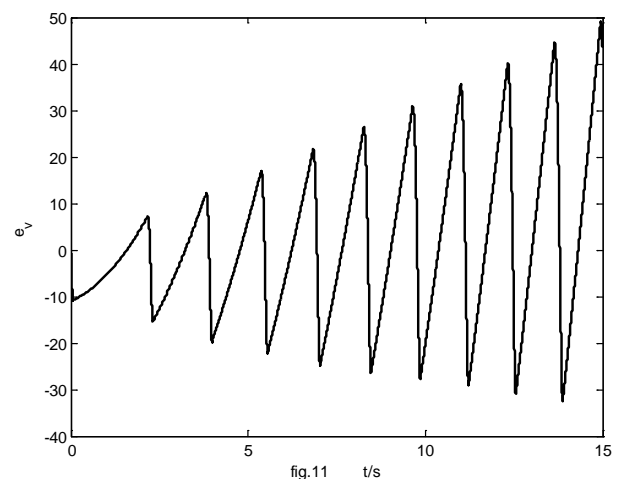

Fig 13 The state of speed error

It is obvious that the speed error is within $50 \mathrm{~m} / \mathrm{s}$, thus the speed control law is effective. Considering that the design is $V^{d}=2391+\Gamma^{*} 0.5 * 15 t$, the acceleration flying ability of hypersonic flight vehicle is tested.

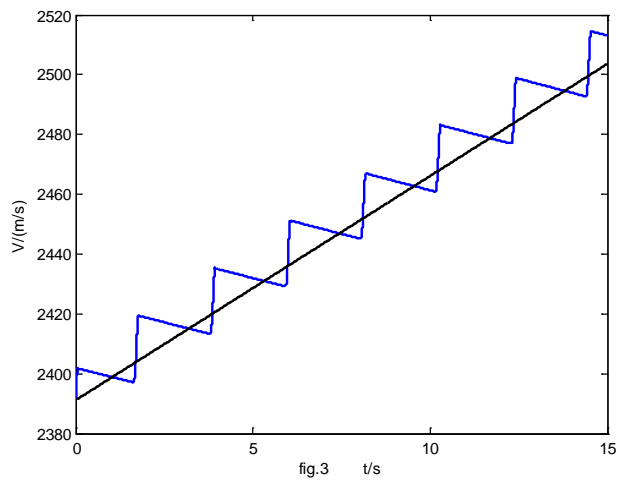

Fig 14 The state of speed error

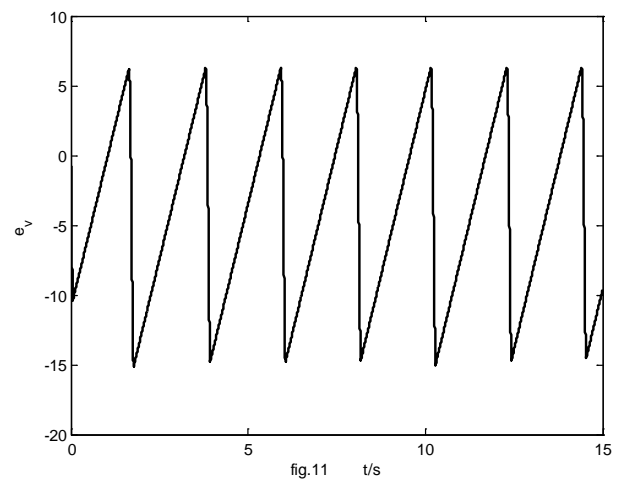

Fig 15 The state of speed error

Visible for acceleration in flight, velocity error can be effectively controlled within $20 \mathrm{~m} / \mathrm{s}$, the hypersonic aircraft is relatively rare.

\section{Conclusion}

Because the engine generates the huge thrust, the coupling effect of engine、 attack angle and elastic form, so the speed control of the hypersonic aircraft is difficult to achieve high precision. The switching law of oil supply is designed based on the PID law and diffusion function in the 
given attack angle and fixed elastic form in this paper. It can be seen from simulation that the proposed control law can realize constant speed and acceleration flight of hypersonic aircraft.

\section{References}

[1]Heller M, Holzapfel F and Sachs G. Robust lateral control of hypersonic vehicles [A]. In: AIAA Guidance, Navigation, and Control Conference and Exhibit [C]. Denver, CO, AIAA 2000-4248.

[2]Appleby B D and Adams N J. Robust estimator design applied to the lateral dynamics of a hypersonic vehicle [A]. In: AIAA Guidance, Navigation, and Control Conference and Exhibit [C]. AIAA-1991-2689.

[3]D.K.Schmidt. Ingegrated Control of Hypersonic Vehicles-A Necessity Not Just a Possibility. AIAA,1993:539-549.

[4]D.K.Schmidt. Optimum Mission Performance and Multivariable Flight Guidance for Airbreathing Launch Vehicles. Journal of Guidance, Control,and Dynamics, 1997,20(6):1157-1164.

[5]Chavez F R and Schmidt D K.Flight dynamics and control of elastic hypersonic vehicles-Modeling uncertainties[A].In:AIAA Guidance, Navigation and Control Conference[C]. Washington,DC, AIAA 94-3629

[6]Chavez F R and Schmidt D K.Uncertainy modeling for multivariable-control robustness analysis of elastic high-speed vehicles[J].Journal of Guidance, Control,and Dynamics,1999,22(1):87-95.

[7]Irene $M$. Gregory . Hypersonic vehicle control law development using $H$ infinity and mu-synthesis. NASA Technical Memprandum. 1994-4526: 1-32.

[8]Bushcek $\mathrm{H}$ and Calise A J.Robust control of hypersonic vehicles considering propulsive and aeroelastic effects[A].In:AIAA Guidance,Navigation and Control Conference[C]. Monterey, CA, AIAA 93-3762.

[9]Buschek $\mathrm{H}$ and Calise A J. Fixed order robust control design for hypersonic vehicles[A].In:AIAA Guidance, Navigation and Control Conference [C]. Washington,DC,AIAA 94-3662

[10]Buschek $\mathrm{H}$ and Calise A J.Hypersonic flight control system design using fixed order robust controllers[A].In:AIAA Guidance,Navigation and Control Conference[C].Chattanooga,TN, AIAA 95-6062

[11]Buschek H, Calise A J. Uncertainty modeling and fixed-order controller design for a hypersonicvehicle model. Journal of Guidance,Control and Dynamics, 1997, 20(1): 42-48.

[12]Heller $\mathrm{M}$ and Sachs G.Flight dynamics and robust control of a hypersonic test vehicle with ramjet propulsion[A].In:AIAA International Space Planes and Hypersonic Systems and Technologies Conference[C].Norfolk,VA,AIAA 98-1521 